\title{
Effects of Directional Permeability Anisotropy on Sweep Efficiency for Five-Spot and Nine-Spot Pattern Flood
}

\author{
Dr. Saad A Balhasan*, Biltayib Misbah and Mohamed A Omer \\ Australian College of Kuwait ACK, Kuwait
}

\begin{abstract}
Water flooding is the dominant fluid injection technique and is frequently applied worldwide secondary recovery process, which involves water injection in the oil formation under high pressure through an injection well to enhance oil recovery of the well(s) of interest. In order to plan implement successful water flooding optimum number of wells and location must be selected. This involves successful and judicious selection of water flood pattern to increase the reserves. This study emphasizes on important and effect of water flood pattern on oil recovery. This paper will also address the effect of the directional permeability on Cartesian and uniform grid. The patterns studied were fivespot and nine-spot pattern and by ignoring the gravity and capillary pressure the designed model will calculate the pressure and the water saturation for all nodes in five and nine spots scheme. The work shows that both pattern schemes produce almost the same tertiary oil under identical reservoir conditions. The nine point's scheme showed smooth pressure and water distribution compared to five point's scheme. A breakthrough time was earlier by $40-50$ days for the nine spot patterns as compared to five spots pattern.
\end{abstract}

\section{Introduction}

The success of a water flood flood project can be predicted from proper selection of water flood patterns. The primary objective is to attain a balance between injection and producer wells within a pattern and minimize oil migration to adjacent patterns and loss into the formation. Unbalanced pattern leads to poor sweep, premature breakthrough and high water cycling Schlumberger [1].

Clark and Robert [2] reported that in 1997, a pilot water flood was initiated in a quiescent part of the reservoir on a 40-acre 5-spot pattern. Four Producers were drilled around a central test water injection well to evaluate the efficacy of water flooding the field. Kuwait Oil Company then initiated an inverted 9-spot pattern water flood to develop the major extent of the field in the most rapid way possible as this entailed the least number of injection lines. The results showed that theoretical sweep efficiency from an inverted 9-spot is inferior to a 5 -spot pattern by approximately $50 \%$ versus $70 \%$. However, to rapidly restore reservoir energy and initiate water flooding operations, a 9-spot pattern was adopted with the eventual goal of down spacing the reservoir to a 5 -spot pattern

Conclusively, Clark and Robert [2] showed that the Kuwait Oil Company was able to optimize water flooding using reservoir management team effective in achieving target reservoir pressures in the major part of the reservoir with the active participation of all team members, subsurface and surface alike. Using this approach, water-cut was flattened with effective decline in wet oil production. According to Moudi [3] further research on the same field showed that applying the new management approach, individual water flood pattern balance was significantly improved and the field wide Voidage Replacement Ration (VRR) become about 1.2.

Barber et al. [4] reviewed nine sandstone and carbonate reservoirs' responses to infill drilling. From the experience in those nine fields, the authors concluded that "the ultimate well density in any given field can be determined only after several years of field performance provide sufficient information on reservoir continuity and recovery efficiencies" [4].

Another paper on this topic is by researchers at Texas A\&M U., [5] who spent several years analyzing the impact of well-spacing reduction on west Texas Clearfork and San Andres water flood performance from various geographic areas of the Permian Basin.

As noted above, many more papers have been published on this topic, but these two papers identify the key well-spacing issues. As both studies conclude, one does not know a priori what the optimal well spacing will be. The technical team must analyze the available data before starting a water flood, and then continue to evaluate the production and injection data to determine the water flood's efficiency and the extent to which infill-well locations should be drilled or patterns realigned.

\section{Procedure}

By consider two type of scheme, five and nine points. In the five points scheme the simulation model will take the effect of the permeability in the main axes $(x, y)$. The water saturation will be calculated by using the pressure values which are solved by the matrix solver.

The same steps has been taken in order to calculate the water saturation in nine point scheme. The directional permeability $k \theta$ has been taken in the consideration to calculate the pressure. The pressure and saturation coefficient are weighted with $1 / 2$ in $(x-y)$ direction and $1 / 4$ in the 45 degree rotated $(\mathrm{v}-\mathrm{w})$.

\section{Solve pressure equation in five point's scheme}

By solve the Diffusivity equation in two dimensions.

$$
\nabla \overline{\bar{K}}\left(\lambda_{t} P_{o}-\left(\lambda_{w} \gamma_{w}+\lambda_{0} \gamma_{o}\right) \nabla D-\lambda_{w} \nabla P_{C W D}\right)+\hat{q}_{t}=\varphi C_{t} \frac{\partial P_{o}}{\partial t}
$$

${ }^{*}$ Corresponding author: Dr. Saad A Balhasan, Australian College of Kuwait ACK, Kuwait, Tel: +965 1828225; E-mail: b.biltayib@ack.edu.kw

Received May 28, 2015; Accepted July 15, 2015; Published July 25, 2015

Citation: Balhasan SA, Misbah B, Omer MA (2015) Effects of Directional Permeability Anisotropy on Sweep Efficiency for Five-Spot and Nine-Spot Pattern Flood. J Pet Environ Biotechnol 6: 238. doi:10.4172/2157-7463.1000238

Copyright: (C) 2015 Balhasan SA, et al. This is an open-access article distributed under the terms of the Creative Commons Attribution License, which permits unrestricted use, distribution, and reproduction in any medium, provided the original author and source are credited. 
Gravity and capillary pressure term are neglecting, so the equation

(1) will reduced to following equation.

$$
\nabla \overline{\bar{K}}\left(\lambda_{t} P_{o}\right)+\hat{q}_{t}=\varphi C_{t} \frac{\partial P_{o}}{\partial t}
$$

Solve equation no. (1) By the finite difference method.

$\frac{\partial}{\partial x}\left(\lambda_{t} K_{x x} \frac{\partial P_{o}}{\partial x}+\lambda_{t} K_{x y} \frac{\partial P_{o}}{\partial y}\right)+\frac{\partial}{\partial y}\left(\lambda_{t} K_{y x} \frac{\partial P_{0}}{\partial x}+\lambda_{t} K_{y y} \frac{\partial P_{0}}{\partial y}\right)+\hat{q}_{t}=\varphi C_{t} \frac{\partial P_{o}}{\partial t}$

Using directional permeability $k \theta$.

$$
\begin{aligned}
& -\left(0.006328 k_{\theta=\pi} \Delta Z \lambda_{t}\right)_{i-1 / 2}\left(P_{O i, j, k}^{n+1}-P_{O i-1, j, k}^{n+1}\right) \\
& +\left(0.006328 k_{\theta=\pi / 2} \Delta Z \lambda_{t}\right)_{j+1 / 2}\left(P_{O i, j+1, k}^{n+1}-P_{O i, j, k}^{n+1}\right) \\
& -\left(0.006328 k_{\theta=3 \pi / 2} k \Delta Z \lambda_{t}\right)_{j-1 / 2}\left(P_{O i, j, k}^{n+1}-P_{O i, j-1, k}^{n+1}\right) \\
& +q_{b}=V R C_{t}\left[\begin{array}{ll}
\frac{P_{O i, j, k}^{n}}{} P_{O i, j, k}^{n} \\
\end{array}\right]
\end{aligned}
$$

Determine the coefficient equations in $(x-y)$ :

$$
\begin{aligned}
& C=\left(T_{x} \lambda_{t}\right) P_{O i, j-1, k}^{n+1}=0.006328^{\star} K_{\theta=3 \pi / 2}{ }^{\star} \Delta z^{\star} \lambda_{t} \\
& D=\left(T_{x} \lambda_{t}\right) P_{O i-1, j, k}^{n+1}=0.006328^{\star} K_{\theta=\pi}{ }^{\star} \Delta z^{\star} \lambda_{t} \\
& F=\left(T_{x} \lambda_{t}\right) P_{O i+1, j, k}=0.006328^{\star} K_{=0}{ }^{\star} \Delta z^{\star} \lambda_{t} \\
& G=\left(T_{x} \lambda_{t}\right) P_{O i, j+1, k}^{n+1}=0.006328^{\star} K_{\theta=\pi / 2}{ }^{\star} \Delta z^{\star} \lambda_{t}
\end{aligned}
$$

Coefficient equation of the source cell.

$E=-\left(C+D+F+G+\frac{\varphi C_{t} \Delta x \Delta y \Delta z}{\Delta t}\right)$

The RHS.

$R_{i j}=-\Delta x \Delta y \Delta z \hat{q}-\frac{\Delta x \Delta y \Delta z}{\Delta t} \varphi C_{t} P_{O i, j, k}^{n}$

\section{Solve the saturation equation in five point's scheme}

By solve the saturation equation by using finite difference method.

$$
\begin{aligned}
& \nabla\left[f_{w} \bar{k} \lambda_{t} \nabla P_{o}\right]+\hat{q}_{w}=\varphi\left[S_{w}\left(C_{\varphi}+C_{w}\right) \frac{d P_{0}}{d t}+\frac{\partial S_{w}}{\partial t}\right] \\
& \frac{\partial}{\partial x}\left(f w \lambda_{t} K_{x x} \frac{\partial P_{o}}{\partial x}\right)+\frac{\partial}{\partial y}\left(f w \lambda_{t} K_{y y} \frac{\partial P_{0}}{\partial y}\right)+\hat{q}_{w}=\varphi\left[S_{w}\left(C_{\varphi}+C_{w}\right) \frac{d P_{0}}{d t}+\frac{\partial S_{w}}{\partial t}\right]
\end{aligned}
$$

Take the finite different for equation (5).

$$
\begin{aligned}
& \left(0.006328 k_{\theta=0} \Delta Z f_{w} \lambda_{t}\right)_{i+1 / 2}\left(P_{O i+1, j, k}^{n+1}-P_{O i, j, k}^{n+1}\right) \\
& -\left(0.006328 k_{\theta=\pi} \Delta Z f_{w} \lambda_{t}\right)_{i-1 / 2}\left(P_{O i, j, k}^{n+1}-P_{O i-1, j, k}^{n+1}\right) \\
& +\left(0.006328 k_{\theta=\pi / 2} \Delta Z f_{w} \lambda_{t}\right)_{j+1 / 2}\left(P_{O i, j+1, k}^{n+1}-P_{O i, j, k}^{n+1}\right) \\
& -\left(0.006328 k_{\theta=3 \pi / 2} k \Delta Z f_{w} \lambda_{t}\right)_{j-1 / 2}\left(P_{O i, j, k}^{n+1}-P_{O i, j-1, k}^{n+1}\right) \\
& +q_{b w}=V R \varphi\left[\frac{S_{w i, j, k}^{n+1}-S_{w i, j, k}^{n}}{\Delta t}\right]+\varphi V R\left[S_{w}\left(C_{\varphi}+C_{w}\right) \frac{P_{o i j k}^{n+1}-P_{o i j k}^{n}}{\Delta t}\right]
\end{aligned}
$$

Solve the Saturation by using the coefficient.

$+\left(0.006328 k_{\theta=0} \Delta Z f_{w} \lambda_{t}\right)_{i+1 / 2}\left(P_{O i+1, j, k}^{n+1}-P_{O i, j, k}^{n+1}\right)$

$$
\begin{aligned}
& -\left(0.006328 k_{\theta=\pi} \Delta Z f_{w} \lambda_{t}\right)_{i-1 / 2}\left(P_{O i, j, k}^{n+1}-P_{O i-1, j, k}^{n+1}\right) \\
& +\left(0.006328 k_{\theta=\pi / 2} \Delta Z f_{w} \lambda_{t}\right)_{j+1 / 2}\left(P_{O i, j+1, k}^{n+1}-P_{O i, j, k}^{n+1}\right) \\
& -\left(0.006328 k_{\theta=3 \pi / 2} k \Delta Z f_{w} \lambda_{t}\right)_{j-1 / 2}\left(P_{O i, j, k}^{n+1}-P_{O i, j-1, k}^{n+1}\right) \\
& +q_{b w}+V R \varphi\left[\frac{S_{w i, j, k}^{n}}{\Delta t}\right]-\varphi V R\left[S_{w}\left(C_{\varphi}+C_{w}\right) \frac{P_{o i j k}^{n+1}-P_{o i j k}^{n}}{\Delta t}\right] * \frac{\Delta t}{\Phi V R}=S_{w i j k}^{n+1}
\end{aligned}
$$

\section{Solve the pressure equation for nine point's scheme}

By solve the pressure equation (1). In nine points we will take in our consideration the directional permeability, and in this case the number of coefficient will be 8 with the source cell.

$$
\begin{aligned}
& +\left(0.006328 k_{=0} \Delta Z_{t}\right)_{1 / 2}\left(P_{O i+1, j, k}^{n+1}-P_{O i, j, k}^{n+1}\right) \\
& -\left(0.006328 k_{\theta=\pi} \Delta Z \lambda_{t}\right)_{i-1 / 2}\left(P_{O i, j, k}^{n+1}-P_{O i-1, j, k}^{n+1}\right) \\
& +\left(0.006328 k_{\theta=\pi / 2} \Delta Z \lambda_{t}\right)_{j+1 / 2}\left(P_{O i, j+1, k}^{n+1}-P_{O i, j, k}^{n+1}\right) \\
& -\left(0.006328 k_{\theta=3 \pi / 2} k \Delta Z \lambda_{t}\right)_{j-1 / 2}\left(P_{O i, j, k}^{n+1}-P_{O i, j-1, k}^{n+1}\right) \\
& +\left(0.006328 k_{\theta=\pi / 4} \Delta Z \lambda_{t}\right)_{i+1 / 2, j+1 / 2}\left(P_{O i+1, j+1, k}^{n+1}-P_{O i, j, k}^{n+1}\right) \\
& \left.-\left(0.006328 k_{\theta=5 \pi / 4} \Delta Z\right)_{t}\right)_{i-1 / 2, j-1 / 2}\left(P_{O i, j, k}^{n+1}-P_{O i-1, j-1, k}^{n+1}\right) \\
& +\left(0.006328 k_{\theta=3 \pi / 4} \Delta Z \lambda_{t}\right)_{i-1 / 2 j+1 / 2}\left(P_{O i-1, j+1, k}^{n+1}-P_{O i, j, k}^{n+1}\right) \\
& -\left(0.006328 k_{\theta=7 \pi / 4} \Delta Z \lambda_{t}\right)_{i+1 / 2, j-1 / 2}\left(P_{O i, j, k}^{n+1}-P_{O i+1, j-1, k}^{n+1}\right) \\
& +q_{t}=V R \varphi C_{t}\left(\frac{P_{O i, j, k}^{n+1}-P_{O i, j, k}^{n}}{\Delta t}\right)
\end{aligned}
$$
$(\mathrm{v}-\mathrm{w})$.

By take the weight $1 / 2$ for the (x-y) and $1 / 4$ for the 45 degree rotated

$$
\begin{aligned}
& C=\left(T_{x} \lambda_{t}\right) P_{O i, j-1, k}^{n+1}=\frac{1}{2}{ }^{\star} 0.006328^{\star} K_{\theta=3 \pi / 2}{ }^{\star} \Delta z^{\star} \lambda_{t} \\
& D=\left(T_{x} \lambda_{t}\right) P_{O i-1, j, k}^{n+1}=\frac{1}{2}{ }^{*} 0.006328^{\star} K_{\theta=\pi} * \Delta z^{\star} \lambda_{t} \\
& F=\left(T_{x} \lambda_{t}\right) P_{O i+1, j, k}^{n+1}=\frac{1}{2}{ }^{\star} 0.006328^{\star} K_{\theta=0}{ }^{\star} \Delta z^{\star} \lambda_{t} \\
& G=\left(T_{x} \lambda_{t}\right) P_{O i, j+1, k}^{n+1}=\frac{1}{2}{ }^{\star} 0.006328^{\star} K_{\theta=\pi / 2}{ }^{\star} \Delta z^{\star} \lambda_{t} \\
& C L=\left(T_{x} \lambda_{t}\right) P_{O i-1, j-1, k}^{n+1}=\frac{1}{4} \star 0.006328^{\star} K_{\theta=5 \pi / 4}{ }^{\star} \Delta z^{\star} \lambda_{t} \\
& D L=\left(T_{x} \lambda_{t}\right) P_{O i+1, j-1, k}^{n+1}=\frac{1}{4} \star 0.006328^{\star} K_{\theta=7 \pi / 4}{ }^{\star} \Delta z^{\star} \lambda_{t} \\
& F R=\left(T_{x} \lambda_{t}\right) P_{O i-1, j+1, k}^{n+1}=\frac{1}{4}{ }^{\star} 0.006328{ }^{\star} K_{\theta=3 \pi / 4}{ }^{\star} \Delta z^{\star} \lambda_{t} \\
& G R=\left(T_{x} \lambda_{t}\right) P_{O i+1, j+1, k}^{n+1}=\frac{1}{4} \star 0.006328^{\star} K_{\theta=\pi / 4} \star \Delta z^{\star} \lambda_{t}
\end{aligned}
$$

The coefficient equation for solve the source cell.

$E=-\left(F+D+G+C+G R+F R+D L+C L+\frac{\varphi C_{t} \Delta x \Delta y \Delta z}{\Delta t}\right)$ and the RHS. 


$$
R_{i j}=-\Delta x \Delta y \Delta z^{*} \hat{q}^{\star}-\frac{\Delta x \Delta y \Delta z}{\Delta t} \varphi C_{t} P_{O i, j, k}^{n}
$$

\section{Solve the saturation equation of the nine point's scheme}

By solve the saturation equation by using finite difference method, take the weight $1 / 2$ for the $(x-y)$ and $1 / 4$ for the 45 degree rotated $(v-w)$.

$$
\begin{aligned}
& +0.5^{\star}\left(0.006328 k_{\theta=0} \Delta Z f_{w} \lambda_{t}\right)_{i+1 / 2}\left(P_{O i+1, j, k}^{n+1}-P_{O i, j, k}^{n+1}\right) \\
& -0.5^{\star}\left(0.006328 k_{\theta=\pi} \Delta Z f_{w} \lambda_{t}\right)_{i-1 / 2}\left(P_{O i, j, k}^{n+1}-P_{O i-1, j, k}^{n+1}\right) \\
& +0.5^{\star}\left(0.006328 k_{\theta=\pi / 2} \Delta Z f_{w} \lambda_{t}\right)_{j+1 / 2}\left(P_{O i, j+1, k}^{n+1}-P_{O i, j, k}^{n+1}\right) \\
& -0.5^{\star}\left(0.006328 k_{\theta=3 \pi / 2} k \Delta Z f_{w} \lambda_{t}\right)_{j-1 / 2}\left(P_{O i, j, k}^{n+1}-P_{O i, j-1, k}^{n+1}\right) \\
& +0.25^{\star}\left(0.006328 k_{\theta=\pi / 4} \Delta Z \lambda_{t}\right)_{i+1 / 2, j+1 / 2}\left(P_{O i+1, j+1, k}^{n+1}-P_{O i, j, k}^{n+1}\right) \\
& -0.25^{\star}\left(0.006328 k_{\theta=5 \pi / 4} \Delta Z \lambda_{t}\right)_{i-1 / 2, j-1 / 2}\left(P_{O i, j, k}^{n+1}-P_{O i-1, j-1, k}^{n+1}\right) \\
& +0.25^{\star}\left(0.006328 k_{\theta=3 \pi / 4} \Delta Z \lambda_{t}\right)_{i-1 / 2 j+1 / 2}\left(P_{O i-1, j+1, k}^{n+1}-P_{O i, j, k}^{n+1}\right) \\
& -0.25^{\star}\left(0.006328 k_{\theta=7 \pi / 4} \Delta Z \lambda_{t}\right)_{i+1 / 2, j-1 / 2}\left(P_{O i, j, k}^{n+1}-P_{O i+1, j-1, k}^{n+1}\right) \\
& +q_{b w}=V R \varphi\left[\frac{S_{w i, j, k}^{n+1}-S_{w i, j, k}^{n}}{\Delta t}\right]+\varphi V R\left[S_{w}\left(C_{\varphi}+C_{w}\right) \frac{P_{o i j k}^{n+1}-P_{o i j k}^{n}}{\Delta t}\right]
\end{aligned}
$$

And,

$$
\begin{aligned}
& +0.5^{\star}\left(0.006328 k_{\theta=0} \Delta Z f_{w} \lambda_{t}\right)_{i+1 / 2}\left(P_{O i+1, j, k}^{n+1}-P_{O i, j, k}^{n+1}\right) \\
& -0.5^{\star}\left(0.006328 k_{\theta=\pi} \Delta Z f_{w} \lambda_{t}\right)_{i-1 / 2}\left(P_{O i, j, k}^{n+1}-P_{O i-1, j, k}^{n+1}\right) \\
& +0.5^{\star}\left(0.006328 k_{\theta=\pi / 2} \Delta Z f_{w} \lambda_{t}\right)_{j+1 / 2}\left(P_{O i, j+1, k}^{n+1}-P_{O i, j, k}^{n+1}\right) \\
& -0.5^{\star}\left(0.006328 k_{\theta=3 \pi / 2} k \Delta Z f_{w} \lambda_{t}\right)_{j-1 / 2}\left(P_{O i, j, k}^{n+1}-P_{O i, j-1, k}^{n+1}\right) \\
& +0.25^{\star}\left(0.006328 k_{\theta=\pi / 4} \Delta Z \lambda_{t}\right)_{i+1 / 2, j+1 / 2}\left(P_{O i+1, j+1, k}^{n+1}-P_{O i, j, k}^{n+1}\right) \\
& -0.25^{\star}\left(0.006328 k_{\theta=5 \pi / 4} \Delta Z \lambda_{t}\right)_{i-1 / 2, j-1 / 2}\left(P_{O i, j, k}^{n+1}-P_{O i-1, j-1, k}^{n+1}\right) \\
& +0.25^{\star}\left(0.006328 k_{\theta=3 \pi / 4} \Delta Z \lambda_{t}\right)_{i-1 / 2 j+1 / 2}\left(P_{O i-1, j+1, k}^{n+1}-P_{O i, j, k}^{n+1}\right) \\
& -0.25^{\star}\left(0.006328 k_{\theta=7 \pi / 4} \Delta Z \lambda_{t}\right)_{i+1 / 2, j-1 / 2}\left(P_{O i, j, k}^{n+1}-P_{O i+1, j-1, k}^{n+1}\right) \\
& +q_{b w}+V R \varphi\left[\frac{S_{w i, j, k}^{n}}{\Delta t}\right]-\varphi V R\left[S_{w}\left(C_{\varphi}+C_{w}\right) \frac{P_{o i j k}^{n+1}-P_{o i j k}^{n}}{\Delta t}\right] * \frac{\Delta t}{\Phi V R}=S_{w i j k}^{n+1}
\end{aligned}
$$

This is the final equation that will use in the simulation model to solve the water saturation at each time steps.

\section{Relative Permeability Equations}

The next following equations have been used to calculate the relative permeability for oil and water at any values of water saturation.

$$
\begin{aligned}
& K_{r w}\left(S_{w}\right)=\dot{K}_{r w}\left(\frac{S_{w}-S_{r w}}{1-S_{r w}-S_{r o w}}\right)^{n_{w}} \\
& K_{\text {row }}\left(S_{w}\right)=\dot{K}_{\text {row }}\left(\frac{1-S_{w}-S_{\text {row }}}{1-S_{r w}-S_{\text {row }}}\right)^{n_{o}}
\end{aligned}
$$

\section{Total Mobility Equation}

This equation has used to calculate the total mobility of oil and water during the flow of two fluids in the model.

$$
\lambda_{t}=\lambda_{w}+\lambda_{o}=\frac{K_{r w}\left(S_{w}\right)}{\mu_{w}}+\frac{K_{r o}\left(S_{o}\right)}{\mu_{o}}
$$

\section{Water Fractional Flow (FW)}

The fractional flow reflects the water front movement in the reservoir and it calculated by the following equation.

$$
f_{w}=\frac{\lambda_{w}}{\lambda_{t}}
$$

\section{Directional Permeability}

The directional permeability is the permeability of the 45 degree $(\mathrm{v}-\mathrm{w})$ when the $(\mathrm{x}-\mathrm{y})$ rotate anticlockwise. The directional permeability will calculated based on the next equation by using the Kmax. and Kmin.

$$
\frac{1}{K_{\theta}}=\frac{\operatorname{Cos}^{2} \theta}{K_{\max }}-\frac{\operatorname{Sin}^{2} \theta}{K_{\min }}
$$

The values of $K_{\max }$ and $K_{\min }$ are 1200 and $100 \mathrm{md}$. Table 1 was prepared based on the above calculation;

\section{Model Description and Reservoir Data}

The 2-D model which used to solve our matrix is consisting of uniform grid $11 \times 11 \times 1$. The fluids will flow from the injector from the center of the mish to the four producers in the corner of the model. The grid considered as homogenous and isotropic. The permeability was different in the both main axes $(x-y)$.

\section{Model Flow chart}

The complete FORTRAN program to compute the pressure and water saturation distribution for nine points scheme is attached in the appendix.

\section{Discussion and Conclusion}

Note that the decrease in production of oil as the layer becomes more saturated by water. Also, the change of oil production rate can be significantly indicated by the changing slope of RF with Time. This

\begin{tabular}{|c|c|}
\hline & \\
\hline $\mathrm{Pi}$ & $2000 \mathrm{psi}$ \\
\hline Porosity & $20 \%$ \\
\hline Injection rate & $400 \mathrm{bbl} / \mathrm{day} / \mathrm{well},-561 \mathrm{cf} /$ day/well \\
\hline$\Delta \mathrm{x}=\Delta \mathrm{y}, \Delta \mathrm{z}$ & $100 \mathrm{ft}, 20 \mathrm{ft}$ \\
\hline Swi & 0.25 \\
\hline Soi & 0.75 \\
\hline Cw & $3.5 \mathrm{E}-61 / \mathrm{psi}$ \\
\hline Co & $1.0 \mathrm{E}-51 / \mathrm{psi}$ \\
\hline C $\Phi$ & $3.0 \mathrm{E}-61 / \mathrm{psi}$ \\
\hline Oil density & $50 \mathrm{lbm} / \mathrm{cf}$ \\
\hline Water density & $62 \mathrm{lbm} / \mathrm{cf}$ \\
\hline Oil viscosity & $4 \mathrm{cp}$ \\
\hline Krw & 0.1 \\
\hline Kro & 0.7 \\
\hline$\alpha=-1.55$, Swx $=0.5$ & $2,3,1.55,0.5$ \\
\hline nw=2, no=3, & \\
\hline
\end{tabular}

Table 1: Contains all the input data used in the model. 
Citation: Balhasan SA, Misbah B, Omer MA (2015) Effects of Directional Permeability Anisotropy on Sweep Efficiency for Five-Spot and Nine-Spot Pattern Flood. J Pet Environ Biotechnol 6: 238. doi:10.4172/2157-7463.1000238

behavior is a function of the relative permeability relationship, and that was very clear when the water saturation increase significantly after the breakthrough. Figures 1 and 2 shows saturation distribution of 9 spot patterns is more uniform as compared to 5 spots patter; this is due to mainly the effect of $\mathrm{K}_{\theta}$.

In five points scheme the water distribution in the mish was move in elliptical ship parallel to the $\mathrm{x}$-axes due to the high permeability. In nine points scheme the water distribution was smooth compared to the five point's scheme and that because of the directional permeability $K \theta$ which create a new distribution of the permeability in the mish. Five snap shot have been taken for pressure and water saturation for each five and nine points. Comparison of patterns indicates that at close well spacing, the nine-spot recovers slightly more oil than the five-spot. Water breakthrough and oil production are accelerated for the nine-spot relative to the production are accelerated for the nine-

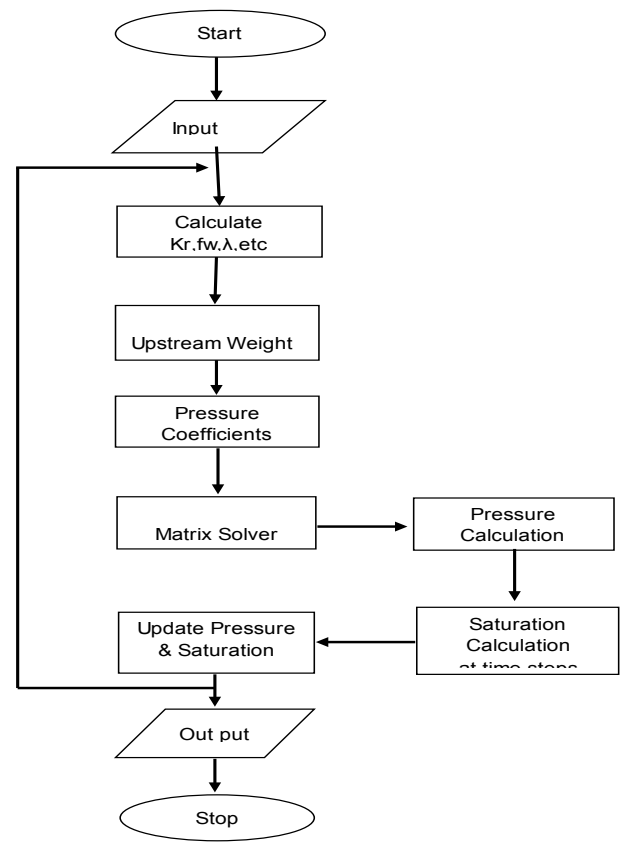

Figure 1: Location map showing the Lake Chad Basin (LCB); the Holocene Mega-Lake Chad as reconstructed by Schuster et al. [22] and the major modern vegetation types [27]. The modern Lake Chad is shown here at its largest extension reached during the past few decades.

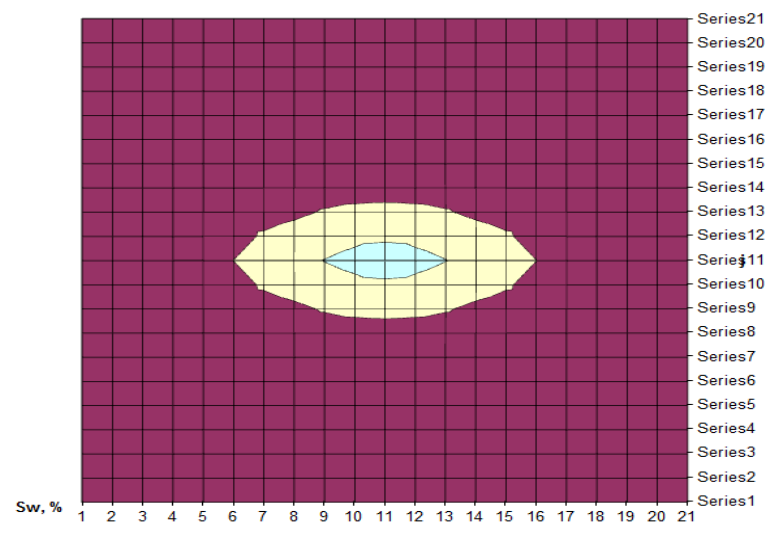

Figure 2: Water saturation of nine spots pattern at 200 days.

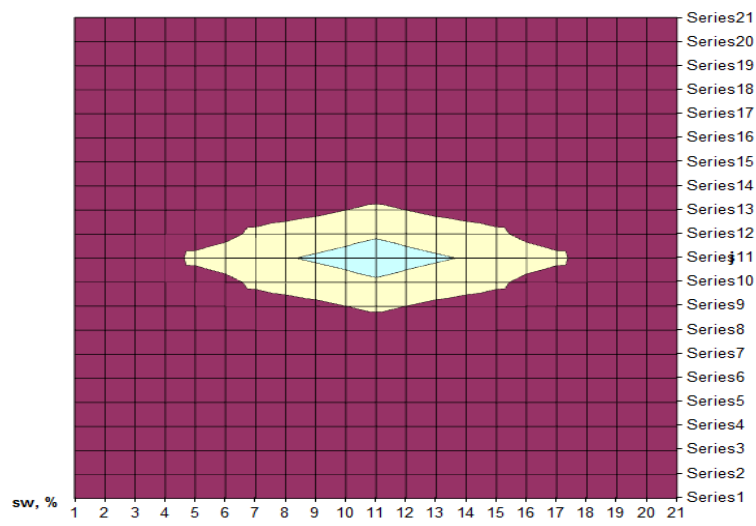

Figure 3: Water saturation of five spots pattern at 200 days.

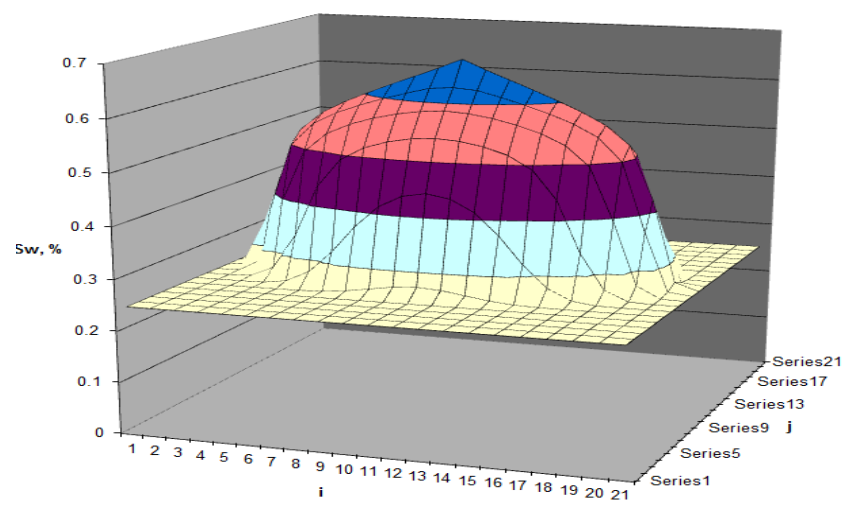

Figure 4: Water saturation on nine spots pattern at 600 days.

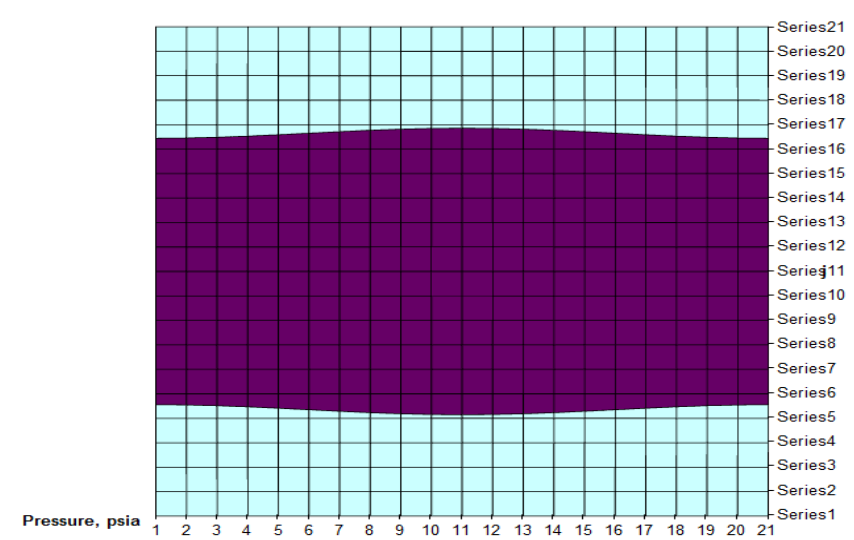

Figure 5: Pressure distribution of nine spots pattern at 4000 days.

spot relative to the five-spot pattern. At larger well spacing, however, oil recovery from the five-spot pattern exceeds that from the nine-spot. The water breakthrough was at 2200 days in the nine points and 2240 at five points. The recovery factor was almost the same $45 \%$ for both the five spots pattern and nine spots pattern.

The fractional flow of water is an important parameter in evaluation of recovery performance of five and nine spot pattern. The fractional flow of water rises abruptly for nine spot pattern as soon as the water 
Citation: Balhasan SA, Misbah B, Omer MA (2015) Effects of Directional Permeability Anisotropy on Sweep Efficiency for Five-Spot and Nine-Spot Pattern Flood. J Pet Environ Biotechnol 6: 238. doi:10.4172/2157-7463.1000238

Page 5 of 5

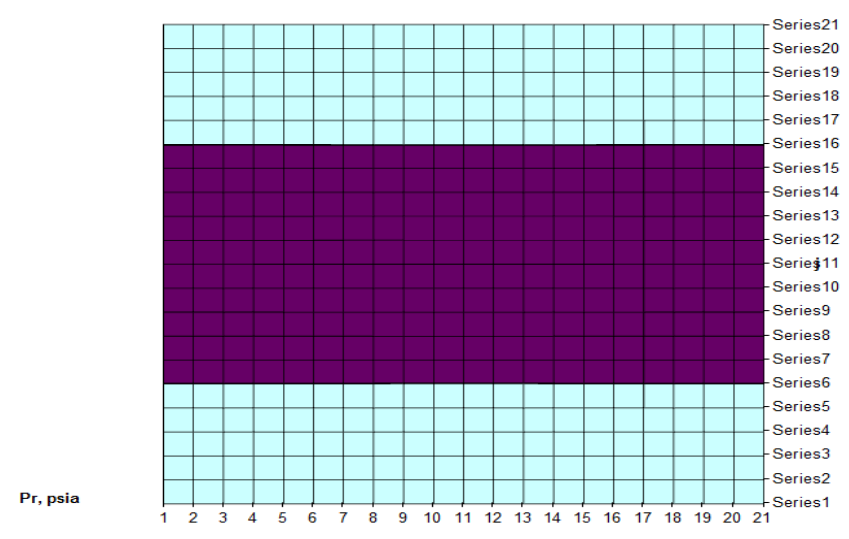

Figure 6: Pressure distribution of five spots pattern at 200 days.

injection is commenced. This might be because of smaller well spacing of water injection and oil production wells. For five spot pattern as the injection and production wells are far away from each other having greater well spacing and less number of water injection wells so fractional flow of water increases steadily in comparison with nine spot pattern. At the later stages water flooding, much of the oil has been recovered so percentage of water flowing in the reservoir is much larger than of oil so the flow rises above $90 \%$ for both the flooding patterns.

\section{References}

1. Three-dimensional, three-phase black oil and compositional streamline simulator (2010) ECLIPSE.

2. Clark Robert A, Lantz JR, Karami H, Al-Ajmi MF(2007) Pattern Balancing and Waterflood Optimisation of a Super giant: Sabiriyah Field, North Kuwait, a case Study.International Petroleum Technology Conference, Dubai, U.A.E.

3. Al-Ajmi MF, Baroon BA, Al-Hamer M, Al-Anzi EHD (2007) Innovative Pattern Balancing and Waterflood Optimization of a Super Giant Carbonate Mauddud Reservoir, Sabiriyah Field, North Kuwait.

4. Barber AH, George CJ, Stiles LH, Thompson BB (1983) Infill Drilling To Increase Reserves-Actual Experience in Nine Fields in Texas, Oklahoma, and Illinois. J Pet Technol 35: 1530-1538.

5. Lu GF, Brimhall RM, Wu CH(1993) Geographical Distribution and Forecast Models of Infill Drilling Oil Recovery for Permian Basin Carbonate Reservoirs. SPE Annual Technical Conference and Exhibition, Houston, Texas. 